\section{Actitudes de los estudiantes y bachilleres acerca de la tesis como modalidad para optar el título de cirujano dentista en la Facultad de Odontología de la Universi- dad Nacional Mayor de San Marcos}

Sfudents and bachelors's attitudes about of the thesis like modality to choose denial surgeon's tille in the san marcos's university

\section{Resumen}

El objetivo del presente estudio fue determinar las actitudes de los estudiantes y bachilleres acerca de la tesis como modalidad para optar el título de Cirujano Dentista en la Facultad de Odontología de la Universidad Nacional Mayot de San Marcos.

Los resultados obtenidos muestran que el mayor porcentaje $(58.4 \%)$ da los bachilleres muestran indiferencia en la actitud hacia la tesis, existe $\tan 21.6 \%$ de aceptación que sumado dan un $\$ 0^{\circ \%}$ en comparacion con solo un $2 \%$ que muestran una actitud de rechazo. Aur cuando exister factures limitantes para reatizar los trabajos de tesis los bachilleres opinan que es satisfactorio el poder realizar trabajo de tesis para optar el título profesional

El mayor porcentaje $(57.65 \%)$ de los estudiantes muestran indiferencia en la actitud hacia la tesis, existe un $10.59 \%$ de aceptación que sumado dan un $68.24 \%$ en comparación con solo un $31 \%$ que muestran una actitud de rechazo. Los factores que limitan la elaboración de tesis de parte de los estudiantes son administrativos, económicos y no los factores académicos, lo que constütuye una fortaleza para mejorar las actitudes hacia la realización de la tesis como medalidad para optar el título profesional.

\section{Abstract}

The present study's objective was to determine the attitudes of the students and bachelors about the thesis as mode stop to choose Dental surgeon's title in Odontology's Faculty of San Marcos's University,

The aftermaths obtunds show than bachelor's bigger percentage they point out $(58.4 \%)$ indifference in the attitude toward the thesis, exists $21,6 \%$. Even when exist bordering factors stop accomplishing the thesis works the bachelors hold that he is satisfactory the could have accomplished thesis work stop to choose the professional title.

The student's bigger percentage they point out indifference in the attitude toward the thesis, exists $10,59 \%$. The factors that limit the thesis elaboration on the side of the students are administrative, economic and no the academic factors, it that a fortress constitutes for the better the attitudes toward the thesis's realization as mode slop to choose the professional title.

\section{Introducción}

La investigación científica es una forma de conocimiento de la realidad caracterizada por su objetividad, precisión y poder predictivo. Además algunos anaden congruencia y originalidad. Existe la creencia que la investigación es solu para científicus y académicos universitarios, y que su metodología es algo difícil y complicada :

Los estudiantes que se encucntran investigando usualmente son percibidos por sus compañeros de años
ARTícUlo ORIGINAL

\section{Mareo Tulio Madrid Chumacero* Juana V. Echeandía Arellano*"}

Departamento Académico de Estomatologita Bjosocial. Faculiaci de Odontología

- Facultad de Medicina Humana

* Universidad Nacional Mayor de San Marcos. Lima, Perrí

E-mail: mmadridc@unmsm.edu.pe

Palabras clave: tesis, bachiller, titulo de cirujano dentista

Key words: thesis, bachelor, dental surgeon's title

último año de estudios se enfrenta a la exigencia de definir un tema de invesw tigación. Los resultados nos dicen que esta elección, en la mayor parte de los casos, no resulta sencilla ${ }^{3}$.

Hacer la tesis involucra no solo un considerable esfuerzo intelectual sino tam bién una constante tensión psicológica.

Hay estudiantes que se deciden por no hacer la tesis porque constituye una pesada carga que es sentida con desagradu y malestar. Algunus no saben que la redacción es la actividad que 
requiere mas esfuerzo y concentración. Escribir bien es difícil y es un proceso de continua revisión que permite alterar, reordenar, añadir, borrar $y$ refinar lo previamente escrito ${ }^{t}$.

La Psicología social atribuye que la conducta de un individuo está en relación a su medio social del cual se orienta sus actitudes. Según Kerlinger la actitud es una predisposición organizada para sentir, percibir y comportarse en cierta forma ante un referente. (Es una categoría, fenómeno, objeto-acontecimiento, conductas c incluso construcciones hipotéticas o un objeto cognoscitivo. Es una estructura estable de creencias que predispone al individuo a comportarse selectivamente ante el referente actitudinales) ${ }^{5}$.A todo lo señalado se puede decir que la actitud es un estado de disposición psicológica, socialmente adquirida y organizada a través de experiencias, de tal manera que el individuo reacciona de una manera caracteristica hacia el objeto, persona o situación específica, siendo esta influenciada por el medio social donde vive y actúa de acuerdo a lo que piensa y siente, poniendo de manifiesto las interaccio-nes positivas o negativas.

Los componentes básicos de las actitudes son el componente cognoscitivo conformado por el conocimiento que el sujeto dispone sobre el objelo de la aclitud, de modo que existe una interrelación mutua, al respecto Pinilla señala que «El conocimiento mutuo origina relaciones progresivamente más complejas" ". Las relaciones sujeto-objeto, constituye un ronocimiento natural en donde cada uno tendrá y mantendrá su rol y su función, correspondiendo al ser humano aplicar su conocimiento científico. El componente afectivo de la actitud está constituido por los aspectos emocionales que acompañan a la motivación, la respuesta emotiva o afectiva necesariamente ticne que estar asociada a una categoría cognosci-tiva del objeto de la actitud, de manera que se va formando en el transcurso de diversos contactos ya sea en forma placentera o desagradable. Esta dirección debe ser educada con el ánimo y en función de la necesidad de estimación y voluntad de poder en la conducta y relaciones inspiradas en actitudes positivas individuales y colectivas. El componente conductual se refiere al acto con que el sujeto reacciona frente al objeto de la actitud traduciéndose en la predisposición conductual del ser humano, al respecto Montesinos y otros señalan que «El componente conductual de una actitud nos ayudará a predecir que conducta mostrará un individuo cuando se enfrente con el objeto de la actitud... ya que no existe consenso respecto al papel de una actitud como activador de la conducta, gran parte de los teóricos están de acuerdo que en la actitud se relaciona de alguna manera con alguna conducta observable. ${ }^{7}$. Este componente sugierc que cada individuo actúa de manera particular ante el objeto en su conjunto en el momento que se enfrenta a él, empero esta conducta tendrá una relación directa asociada con la afectividad, de manera que si el individuo tiene una actitud negativa o positiva hacia el objeto, la respuesta lógica cuando se enfrente al el también será de rechazo o aceptación a la misma. Esta conducta del individuo o grupo tendrá entonces que reflejarse en el ambiente social (costumbres, ideas, condiciones de trabajo y vida, etc.) entonces éstas conductas estarán implícitas en que descansan bajo la base sólida de la realidad social que influye en la educación y formación de cada individuo.

Los tipos de actitudes son la actitud de aceptación en el sentido amplio del término, implica la correspondencia de un aspecto esencial del sujeto hacia un determinado objeto enmarcado dentro de una aceptación positiva de la misma, donde el individuo podrá desenvolverse y establecer una relación de responsabilidad consigo mismo y con el objeto de dicha actitud, en ese sentido Pinilla refiere que «la actitud es positiva cuando se organiza la propia conducta en pro del establecimiento de una relación en la que predomina la aceptación, la estimación, el apoyo, ayuda y la cooperación. El sentido de comprensión viene a scr la predisposición para la comunicación y para la interacción humana positiva" ${ }^{8}$. Los sentimientos de identificación con otra persona u objeto (aceptación) se llega a producir cuando se disuelven las barreras que puedan existir entre ambos, tal es así que compartirá sus valores, tomándolos como propios. En una actitud favorable también se percibe los sentimientos de responsabilidad que consisten en tomar parte activa y asumir un compromiso frente a una necesidad, es importante para la formación de una actitud responsable, el conocimiento cabal de la importancia de satisfacer una necesidad y las disponibilidad para un acercamiento con el objeto o situación y llevar a cabo tareas $n$ actividades que tiendan a solucionar el problema.

La actitud de rechazo, en tanto cuando una actitud es negativa, se presen ta el recelo que aleja a la persona del objeto, el recelo es opuesto a la cooperación por cl distanciamiento que suscita y genera el mecanismo de defensa en la persona.

El ser humano para anticiparse y deterrer su ansiedad provocada por su estímulo frustrante y si este es prolongado entonces modifica la conducta de la persona y altera su actitud su motivación, su memoria, su pensamiento y su acción humana, esta ronducta así modificada será representada por la persona que determinará su actitud de rechazo y en adelante actuará con sentimientos de evasión pretextando motivos de poca importancia.

Las características de las actitudes son las siguientes: las actitudes son aprendidas. La actitud no es innata, se aprende de la experiencia y aprendizaje social en forma voluntaria e involuntaria. Las actitudes son dinámicas. La actitud es susceptible de modificarse o una vez formadas pueden perdurar en el sujeto. Las actitudes se interrelacionan. La actitud constituye una relación entre personas o entre una persona y el objeto, es así como se van formando en relación a algo. La intensidad de la actitud nos indica el aspecto emocional de la actitud, la que determina el grado de convicción de una persona hacia el objeto. La dirección de la actitud implica el modo de sentir de una persona hacia el objeto, así un estudiante o un bachiller puede ante una situación de elaboración de tesis para optar el título, su actitud hacia la misma será de aceplación o rechazo, así también puede decirse favorable o desfavorable. La intensidad así como la dirección conforman el componente afectivo de las actitudes.

El artículo $\mathrm{N}^{\circ} 10^{\circ}$ del reglamento para optar el titulo de cirujano dentista en la facultad de odontología de la UNMSM. Dice que para optar el Titulo Profesional de Cirujano Dentista se requiere: Haber completado el Currículo de la Facultad de Odontología, poseer el Grado Académico de Bachiller en Odontología y Sustentar y aprobar una Tesis de Investigación 
ò la presentación de una Monografía de un Caso Clínico y su respectiva sustentación. En años recientes se ha desarrollado una nueva modalidad para optar el título profesional que consiste en la aprobación de un curso de titulación para bachilleres lo que hace que gran porcentaje de estudiantes y bachilleres decidan no hacer la tesis para optar el título profesional. El presente trabajo permite conocer cuai es la actitud de los estudiantes y bachilleres acerca de la tesis además de establecer las debilidades y fortalezas para el desarrollo de la tesis. ${ }^{9}$

\section{Objetivo}

Determinar las actitudes de los estudiantes y bachilleres acerca de la tesis como modalidad para optar el título de Cirujano Dentista en la Facultad de Odontología de la Universidad Nacional Mayor de San Marcos

\section{Justificación}

1. Conocer los obstáculos y limitaciones que enfrentan los estudiantes al decidirse hacer la tesis.

2. Conocer limitaciones en el proceso de claboración de tesis

3. Incrementar el número de alumnos que opten la modalidad de titulación con la sustentación de tesis

4. Fortalecer la metodología de la investigación como estrategia que conlleve a la motivación de los estudiantes y con ello mejorar la formación académica en investigación conducente a elaborar proyectos de tesis.

\section{Material y métodos}

Para la ejecución del presente estudio de investigación se utilizó el tipo de estudio descriptivo transversal. El área de estudio considerado fue la Facultad de Odontología de la UNMSM. La población de esludio fueron 85 alumnos del quinto año matriculados en la asignatura Clínica Integral del Adulto y 125 bachilleres matriculados en el curso de titulación del año 2001 y 2002.

El instrumento de medición de actitudes estuvo basado en la escala tipo Likert.

Para la recopilación de la información se solicitó la autorización a nivel de dirección de la Escuela Académico

Profesional de Odontología de la UNMSM, igualmente se coordinó con los profesores responsables de cada curso, obteniéndose así la relación de alumnos con asistencia regular por cada asignatura profesional . El estudio se realizó de Diciembre 2001 a Diciembre del 2002.Previa a la aplicación de la escala se impartió las instrucciones pertinentes y se resolvió las dudas en cada caso que lo requería cada unidad muestral, para la aplicación del instrumento se utilizó un tiempo de 10 a 15 minutos como promedio.

El prucesamiento de dato una vez de haber terminado de recolectar los datos, se procedió a la clasificación y codificación de los mismos obteniendo una base de datos utilizando el programa Statistical Package Social Sciencies (SPSS). Para el procesamiento del cuestionario se utilizó la escala de Likert, cuya puntuación por ítems corresponde a las respuestas dadas por los estudiantes y bachilleres de odontología, asignándose el siguiente puntaje.

\begin{tabular}{lc}
\hline Afirmaciones Positivas. & Valor de la Escala \\
Completamence de acuerdo & 4 \\
De acuerdo & 3 \\
Indeziso & 2 \\
En desacieido & 1 \\
Completamente en desacuerdo & 0
\end{tabular}

Afirmaciones Negativas Valor de la Escala Completamente de acuerdo

De acuerdo

Indeciso

En desacuerds

Completamente en desacuerdo

0

1

2

3

Luego se procedió a oblener el punitaje de cada unidad muestral, una vez oblenido la totalidad de los puntajes se hizo una distribución de frecuencias y demás estadisticas descriptivas como media, desviación estándar, sumatoria entre otros.

luego se aplicó la prueba de distribución normal obteniéndose la escala de valores con su respectiva puntuación (intervalo) cuyos puntajes obtenidos nos indican la Aceptación o Rechazo de la actitud de los estudiantes y bachilleres hacia la tẹsis

Se usaron pruebas de $t$, expresándose los resultados como valor $\mathrm{p}$.

\section{Resultados}

a) Actitudes hacia la tesis de los bachilleres

En relación a las actitudes de los bachilleres acerca de la tesis como modalidad para optar el título de cirujano dentista en la facultad de odontología de la universidad nacional ma yor de san marcos, $73(58.4 \%)$ tienen una actitud de indiferencia , 27 (21.6\%) tienen una actitud de aceptación y 25 $(20.0 \%)$ asumen una actitud de rechazo. (Ver cuadro $\mathrm{N}^{\circ} 1$ )

\section{Prueba de distribución normal}

$\mathrm{a}=$ Promedio $-0.75^{*}$ Desv. Estándar

$a=153.48-0.75^{\times} 19.86=138.585$

$\mathrm{b}=$ Promedio $-0.75^{*}$ Desv. Estándar

$\mathrm{b}=153.48+0.75^{*} 19.86=168.375$

b) Actitudes hacia la tesis de los estudiantes

En relación a las actitudes de los estudiantes acerca de la tesis como modalidad para optar el título de cirujano dentista en la facultad de odontología de la universidad nacional mayor de san marcos, $49(57.65 \%)$ tienen una actilud de indiferencia , 9 (10.59 $\%$ ) (ienen una actitud de aceptación y $27(31.76 \%)$ asumen una actitud de rechazo. (ver cuadro $\mathrm{N}^{\circ} 2$ )

c) Factores que influyen en los estudiantes en el desarrollo de la tesis

Respecto a los ítems de respucstas, los factores que mas influyen negativamente en los estudiantes tenemos a que no existen muchas facilidades para realizar trabajos de investigación, no existen muchas facilidades para realizar trabajos de investigación, el nivel económico de los estudiantes no permite realizar la tesis, los trabajos

Estadisticos Descriptivos

\begin{tabular}{|ll|}
\hline Mínimo & 98 \\
\hline Máximo & 204 \\
\hline Sumatoria de puntajes & 19185 \\
\hline Error estándar de la media & 1.78 \\
\hline Promedio & 153.48 \\
\hline Desviación estándar & 19.86 \\
\hline Varianza & 394.365 \\
\hline
\end{tabular}


Cuadro $\mathrm{N}^{\circ} 1$. Actitudes de los bachilleres acerca de la tesis como modalidad para optar el título de cirujano dentista en la Facultad de Odontología de la Universidad Nacional Mayor de Aan Marcos. Año 2002

\begin{tabular}{|l|c|c|c|}
\hline ACTITUD & PUNTUACION & $\mathrm{N}^{\circ}$ & $\%$ \\
\hline Rechazo & $98-139$ & 25 & 20.0 \\
\hline Indiferencia & $140-168$ & 73 & 58.4 \\
\hline Aceptación & $169-204$ & 27 & 21.6 \\
\hline Total & & 125 & 100.00 \\
\hline
\end{tabular}

Estadisticos Descriptizos de los Estridiantes

\begin{tabular}{|ll|}
\hline Mínimo & 30 \\
\hline Máximo & 150 \\
\hline Promedio & 98.56 \\
\hline Desviación estándar & 7.86 \\
\hline Varianza & 61.78 \\
\hline
\end{tabular}

\section{Prueba de distribución normal}

$\mathrm{a}=$ Prom $-0.75^{*}$ Desv. Estándar
$\mathrm{a}=98.56-0.75^{* 7.86}=92.665$

$\mathrm{b}=$ Prom $-0.75^{*}$ Desv. Estándar

$\mathrm{b}=153.48+0.75^{*} 7.86=104.455$

Cuadro $\mathrm{N}^{\circ}$ 2actitudes de los estudiantes acerca de la tesis como modalidad para optar el título de cirujano dentišta en la Facultad de Odontología de la Inniversidad Nacional Mayor de San Marcos. Año 2002.

\begin{tabular}{|l|c|c|c|}
\hline ACTITUD & PUNTUACION & $\mathrm{N}^{\circ}$ & $\%$ \\
\hline Rechazo & $105-150$ & 27 & 31.76 \\
\hline Indiferencia & $93-104$ & 49 & 57.65 \\
\hline Aceptación & $30-92$ & 9 & 10.59 \\
\hline Total & & 85 & 100.00 \\
\hline
\end{tabular}

de investigación no son importantes en la formación académica, el tiempu empleado en la realización de un trabajo de tesis es una pérdida.

Asimismo hay que resaltar que existen factores positivos tales como Todos los profesores están capacitados para brindar adecuada orientación en los trabajos de tesis, La mayoría sabe leer criticamente informes de investigación, La orientación recibida de parte de los profesores en trabajos de investigación es bastante ilustrativa, El horario de estudios no interfiere en el momento de realizar la tesis y La formación académica confiable de los profesores permite guiar y orientar al alumno en el trabajo de tesis. (Ver cuadro $\mathrm{N}^{\circ} 3$ ).

d) Factores que influyen en los bachilleres en el desarrollo de la tesis

Respecto a los items de respuestas, los factores que mas influyen negativamente en los bachilleres tenemos a que se hace necesaria una mayor orientación sobre la realización de tesis, debería existir un reconocimiento especial para la rnejor tesis, no existen facilidades para
Cuadro $\mathrm{N}^{\circ}$ 3. Factores que mas influyen en los estudiantes de la Facultad de Odontología de la Universidad Nacional Mayor de San Marcos en el desarrollo de la tesis. Año 2002

1. No existen muchas facilidades para realızar trabajos de investigación

2. No existen muchas facilidades para realizar trabajos de investigación

3. El nivel económica de los estudiantes no permite realizar la tesis

4. Los trabajos de investigación no son importantes en la formación acaciérnica

5. Todos los profesores están capacitados para brindar aciecuada orientación er. los tráoajos de tesis.

6. La mayoria sabe leer criticamente informes de investigación

7 La orientación recibida de parte de los profesores en trabajos ce investigación es bastarte ilustrativa.

8. El horario de estudios no interfiere en el momento de realizar la tesis

9. La formación académica confiable de los profesores permite guiar y orientar a alumno en el trabajo de tesis

10.El tiempo empleado en la realización de un trabajo de tesis es una pérdida

\section{Discusión}

De acuerdo con los resultados obtenidos ya se conocen los obstáculos y limitaciones que enfrentan los estudiantes al decidirse hacer la tesis. Y por lo tanto es urgente atender a los requerimientos. Así mismo conocidas las li mitaciones en el proceso de elaboración de tesis se hace necesario establecer premios, motivaciones para el logro de los objetivos que cada vez los alumnos se decidan a hacer tesis de esta manera se puede incrementar el número de alumnos que opten la modalidad de titulación con la sustentación de tesis. Asimismo es necesario fortalecer la metodología de la investigación como estrategia que ronlleve a la motivación de los estudiantes y con ello mejorar la formación académica en investigación conducente a elaborar proyectos de tesis.

Teniendo en cuenta que de acuerdo a la teoría elaborada por la Psicología social hay que actuar sobre los componentes básicos de las actitudes en nuestros estudiantes, especialmente en los componentes afectivo y conductual, para lograr el paso de la indiferencia a la aceptación teniendo en cuenta que las actitudes son aprendidas, se aprende de la experiencia y aprendizaje social y son susceptibles de modificación. 
Cuadro $N^{\circ}$ 4. Factores que mas influyen en los bachilleres de la racultad de Udontología de la Universidad Nacional Mayor de San Marcos en el desarrollo de la tesis. Año 2002. En el desarrollo de la tesis

\begin{tabular}{|l|}
\hline 1. Se hace necesaria una mayor orientación sobre la realización de tesis \\
\hline 2. Debería existir un reconocimicnto cspecial para la mejor tesis \\
\hline 3. No exislen facilidades para realizar tesis \\
\hline 4. En la Universidad no existen material suficiente bibliográfico \\
\hline 5. Las tesis son importantes en la formación profesional \\
\hline 6. Los trabajos monográficos requieren menos esfuerzu en su realización que las tesis \\
7. Desalienta saber que las conclusiones a las que se llegan en las tesis no son tomadas \\
en cuenta \\
\hline 8. Realizar trabajos de tesis es agradable \\
\hline $\begin{array}{l}\text { 9. Las calificaciones que dan los profesores a los trabajos de tesis no retribuyen el } \\
\text { esfuerzo empleado en su elaboración }\end{array}$ \\
\hline i0. No sahe escoger un tema apropiado para ser investigado \\
\hline
\end{tabular}

Estadísticos de grupo

\begin{tabular}{|l|c|c|c|c|c|}
\hline & $\begin{array}{l}\text { Variables de } \\
\text { agrupación }\end{array}$ & $\mathrm{N}$ & Media & $\begin{array}{c}\text { Desviación } \\
\text { típ. }\end{array}$ & $\begin{array}{c}\text { Errot típ. de } \\
\text { la media }\end{array}$ \\
\hline $\begin{array}{l}\text { Contrastar } \\
\text { variables }\end{array}$ & 1.00 & 50 & 3.1296 & .90986 & .12867 \\
& 2.00 & 50 & 3.0220 & .77144 & .10910 \\
\hline
\end{tabular}

Cuadro $\mathrm{N}^{\circ}$ 5. Comparación de medias para dos muestras con datos independientes. Prueba de muestras independientes.

\begin{tabular}{|c|c|c|c|c|c|c|c|c|c|}
\hline \multicolumn{3}{|c|}{$\begin{array}{l}\text { Prueba de Levene para la } \\
\text { igualdad de varianzas }\end{array}$} & \multicolumn{7}{|c|}{ Prueba $T$ para la igualdad de medias } \\
\hline & $\mathrm{F}$ & Sig. & $\mathrm{t}$ & $\mathrm{gl}$ & $\begin{array}{l}\text { Sig. } \\
\text { (bila- } \\
\text { teral) }\end{array}$ & \begin{tabular}{|c|} 
Dife- \\
rencia \\
de \\
medias
\end{tabular} & $\begin{array}{l}\text { Error típ. } \\
\text { de la } \\
\text { diferen- } \\
\text { cia }\end{array}$ & $\begin{array}{r}95 \% \text { In } \\
\text { de con } \\
\text { par } \\
\text { difer }\end{array}$ & $\begin{array}{l}\text { tervalo } \\
\text { afianiza } \\
\text { a la } \\
\text { encia }\end{array}$ \\
\hline & & & & & & & & Inferior & Superion \\
\hline $\begin{array}{l}\text { asumien- } \\
\text { do } \\
\text { va rianzas } \\
\text { iguales }\end{array}$ & .281 & .597 & .638 & 98 & .525 & .10760 & .16870 & -.22718 & .44238 \\
\hline $\begin{array}{l}\text { No } \\
\text { asumien- } \\
\text { do } \\
\text { varianzas } \\
\text { iguales }\end{array}$ & & & .638 & 95.447 & .525 & .10760 & .16870 & -.22729 & .44249 \\
\hline
\end{tabular}

\section{Conclusiones}

- Aun cuado los Bachilleres indican que existen factores limitantes para realizar los trabajos de tesis, también opinan que le es salisfactorivel poder realizar trabajos de tesis para optar el título profesional.

- Si bien es cierto el mayor porcentaje de los bachilleres evidencia indiferencia en la actitud hacia la tesis, existe un porcentaje de aceptación cuya suma es mayor que el porcentaje de rechazo

- Entre factores limitantes para realizar los trabajos de tesis que enfrentan los estudiantes están relacionados con factores administrativos y económicos y no así con factores académicos.

- Si bien es cierto el mayor porcentaje de los estudiantes evidencia indiferencia en la actitud hacia la tesis, existe un porcentaje de aceptación cuya suma es mayor que el porcentaje de rechazo

\section{Recomendaciones}

- Que la Facultad de Odontología a través de la $\mathrm{EAPO}$, considere las conclusiones de la investigación, para un anălisis en relación a los contenidos actitudinales que se relacionan con la elaboración de la tesis.

- Conocidos los factores que afectan y/ o ayudan al desarrollo de la tesis establecer un programa de intervención que permita a los estudiantes y bachilleres decidir hacer tesis como modalidad para optar el lítulo profesional.

- Desarrollar asignaturas de la elaboración de tesis, seminarios, programas de capacitación y actualización en relación a la elaboración de tesis, de manera que permita afianzar las actitudes favorables y modificar las actitudes desfavorables de los estudiantes y bachilleres.

\section{Referencias}

1.Portocarrero Suárez, Felipe. Cómo hacer un trabajo de investigación. Centro de investigaciones (CIUP) Universidad del Paćfico. Segunda edición. 1989.Perú.

2.Watson, Ceorge, Writing a Thesis: a guide tolong essays and dissertations, p 39 England, 1988.

3.Agnes G. Resler. Evaluación de Actitudes. Programa de personal de salud. Taller sobre evaluación del aprendizaje y sistematización de la enseñanza. Evaluación de actitudes. Taller sobre evaluación del aprendizaje y sistematización de la enseñanza. Programa de Personal de Salud. OPS/ OMS Ref. HSMD /EV/10.

4.Oscar Soria. Docencia de la Investigación en la Universidad. Separata.

5.Kerlinger, Fred. Investigaciones del comportamiento. Mexico, Ed. Interamericana, 1982, lag. 346.

6.Pinilla, Antonio. La integración Humana, Lima: Ed offet, 1970, Pag.90.

7.Motesinos, Gladis y Otros. Psicología Social. Perú. Ed. Biblioteca, 1986́. Pag. 35.

8.Pinilla, Antonio. Psicología Social LimaPerú.1cra.ed. Ed. Universidad de lima.1969, Pag.103.

9.Facultad de Odontología de la Universidad Nacional Mayor de San Marcos. Reglamento para optar el Titulo de Cirujano Dentista en la Facultad de Odontología de la UNMSM. 